\title{
Intramuscular injection of bone marrow mononuclear cells contributes to bone repair following midpalatal expansion in rats
}

\author{
XIAOXIA CHE $^{1 *}$, JIE GUO $^{2 *}$, XIANGDONG LI $^{1}$, LVE WANG $^{3}$ and SILONG WEI ${ }^{1}$ \\ ${ }^{1}$ Department of Orthodontics, School of Stomatology, Capital Medical University, Beijing 100050; \\ ${ }^{2}$ Department of Orthodontics, School of Stomatology, Shandong University, \\ Shandong Provincial Key Laboratory of Oral Biomedicine, Jinan, Shandong 250012; ${ }^{3}$ Department of Microbiology, \\ College of Life Science and Technology, Beijing University of Chemical Technology, Beijing 100029, P.R. China
}

Received December 15, 2014; Accepted October 19, 2015

DOI: $10.3892 / \mathrm{mmr} .2015 .4578$

\begin{abstract}
Healing from injury requires the activation and proliferation of stem cells for tissue repair. Previous studies have demonstrated that bone marrow is a central pool of stem cells. The present study aimed to investigate the route undertaken by bone marrow mononuclear cells (BMMCs) following BMMC transplantation by masseter injection in a rat model of midpalatal expansion. The rats were divided into five groups according to the types of midpalatal expansion, incision and BMMC transplantation. Samples of midpalatal bone from the rats in each group were used for histological and immunohistochemical assessments to track and evaluate the differential potentials of the transplanted BMMCs in the masseter muscle and midpalatal bone. Bromodeoxyuridine was used as a BMMC tracing label, and M-cadherin was used to detect muscle satellite cells. The BMMCs injected into the masseter were observed, not only in the masseter, but also in the blood vessels and oral mucosa, and enveloped the midpalatal bone. A number of the BMMCs transformed into osteoblasts at the boundary of the neuromuscular bundle, and were embedded in the newly formed bone during midpalatal bone regeneration. The results of the present study suggested that BMMCs entered the circulation and migrated from muscle to the bone tissue, where they were involved in bone repair. Therefore, BMMCs may prove useful in the treatment of various types of cancer.
\end{abstract}

Correspondence to: Dr Xiaoxia Che, Department of Orthodontics, School of Stomatology, Capital Medical University, 4 Tiantan Xili, Dongcheng, Beijing 100050, P.R. China

E-mail: xiaoxiache2014@163.com

*Contributed equally

Key words: bone marrow mononuclear cells, intramuscular injection, midpalatal expansion, bone repair

\section{Introduction}

Skeletal injury is one of the most prevalent problems observed clinically, which impairs the daily activities of the patients (1). Bone repair is regulated by a highly coordinated series of molecular, cellular and tissue events; and the recruitment, proliferation and accumulation of stem cells at the site of injury are the essential aspects of the repair process (2). Immature stem cells differentiate into dynamic osteoblasts in order to restore bone tissues (3). Bone injury healing also critically depends on the growth of blood vessels, not only for nutrient supply, but also for the influx of osteoblasts $(4,5)$. Previous evidence has indicated that physiological bone remodeling occurs in close proximity to blood vessels, and these vessels carry perivascular stem cells, which then differentiate into osteoblasts (5).

The marrow stroma is a complex tissue, which contains cells that are required for the lineage commitment of hematopoietic and non-hematopoietic cells and can differentiate into various types of mesenchymal and non-mesenchymal cell (6). Bone marrow mononuclear cells (BMMCs) include several types of marrow stem cells, including hematopoietic stem cells, mesenchymal stem cells, endothelial progenitor cells and other precursor stem cells (7). Following trauma, these cells secrete cell growth factors, which promote tissue differentiation of the transplanted BMMCs, leading to repair of the injured organs and restoration of organ function (8-10). In preclinical and clinical trials, bone marrow-derived stem cells have been successfully applied for bone and cartilage regeneration following osteoporotic fracture and arthritis $(11,12)$.

Although studies have suggested that homing of native stem cells to injured tissue and their subsequent involvement in the regenerative process is a natural healing response (13-15), the mechanism underlying the function of these stem cells in bone repair remains to be elucidated. In the present study, a rat model of midpalatal incision and expansion was constructed and used to investigate the mechanism underlying the movement of transplanted BMMCs into the circulation and how they become stationed at the injury site for bone repair. The results may provide further understanding of bone injury healing by stem cells. 


\section{Materials and methods}

Animals and grouping. A total of 15 male, 8-week-old Sprague-Dawley rats (Vital River Laboratory Animal Technology Co., Ltd., Beijing, China), with a mean weight of $208.36 \pm 7.32 \mathrm{~g}$, were used in the present study. The rats were housed in an International Standards Organization (ISO) class 7 room with $50 \%$ relative humidity. The rats underwent a reversed 12:12 h light/dark cycle and were maintained at $22-25^{\circ} \mathrm{C}$ in an atmosphere containing $5 \% \mathrm{CO}_{2}$. They were given $5 \mathrm{~g}$ feedstuff and $11 \mathrm{ml}$ per $100 \mathrm{~g}$ body weight of water daily. All groups of animals were housed together with a different marker. All rats were treated according to the ethical regulations defined by the Ethics Committee of Capital Medical University (Beijing, China).

The rats were divided into five groups, with three rats in each group, as follows: Control group (Inc), in which the midpalatal suture was sectioned without expansion or BMMC transplantation; expansion group (Exp), in which the rats underwent midpalatal expansion for 2 weeks with incision, but no BMMC transplantation; expansion and transplantation group (EaT), in which the rats underwent midpalatal expansion for 2 weeks with incision and BMMC transplantation; expansion and relapse group (ExR), in which the rats underwent midpalatal expansion for 2 weeks with incision, but no BMMC transplantation, followed by removal of the expansion appliance $(0.45 \mathrm{~mm}$ stainless steel AJ Wilcock Australian Wire; AJ Wilcock PTY., Ltd., Whittlesea, Australia) and observation of palatal changes during relapse; expansion/transplantation/relapse group (EtR), in which the rats underwent midpalatal expansion for 2 weeks with incision and BMMC transplantation, prior to removal of the expansion appliance and sacrifice of the rats 2 weeks later in order to observe palatal changes during relapse. The rats were sacrificed by cervical dislocation. None of the groups of rats experienced a significant loss of body weight during the fed period. At the beginning of rapid suture expansion, food intake was disturbed, however, the body weight of the rats recovered afterwards. Samples of midpalatal tissues were obtained from these rats in each group, which were used for further histological and immunohistochemical analyses.

Expansion of the midpalatal suture. The rats were subjected to midpalatal expansion, as described in a previous study (16). The distal ends of the expansion appliances were placed into the inter-proximate space between the second and third molars, and the appliances were activated through the ends of the compression helices to exert an initial expansion force of $150 \mathrm{~g}$. A $1.5 \mathrm{~cm}$ anteroposterior mid-sagittal incision was made following appliance placement. The expansion appliances were activated once every other day (150 g each time) in order to obtain the effects of midpalatal expansion. The duration of midpalatal expansion was two weeks.

BMMC culture and transplantation. Bone marrow aspirate is considered to be the most accessible and enriched source of mesenchymal stem cells due to the efficient isolation of multi-potential cells from this tissue (17). Mouse BMMCs were harvested and cultured using a previously described method with modifications (18). Briefly, a 2-month-old male BALB/C mouse (Vital River Laboratory Animal Technology
Co., Ltd.) was chosen as the donor of BMMCs. The mouse was housed in an ISO class 7 room with 50\% relative humidity. The mouse underwent a reversed 12:12 h light/dark cycle and was maintained at $22-25^{\circ} \mathrm{C}$ in an atmosphere containing $5 \% \mathrm{CO}_{2}$, and was given $4 \mathrm{~g}$ feedstuff and $5 \mathrm{ml}$ per $100 \mathrm{~g}$ body weight of water daily. BMMCs were obtained by flushing the femur of the mouse with Dulbecco's modified Eagle's medium (DMEM)/F12 (Invitrogen; Thermo Fisher Scientific, Inc., Waltham, MA, USA), and centrifuged at $400 \mathrm{x}$ g for $10 \mathrm{~min}$ to remove adipose tissue. The cell pellet was suspended in $5 \mathrm{ml}$ chilled Hanks' balanced salt solution and layered over $8 \mathrm{ml}$ Percoll solution $(1.073 \mathrm{~g} / \mathrm{ml}$ ) (both GE Healthcare Life Sciences, Chalfont, UK). Following centrifugation at $800 \mathrm{x}$ g for $30 \mathrm{~min}$, the mononuclear cell layer was removed from the interface, suspended in DMEM/F12 supplemented with $20 \%$ fetal bovine serum, $50 \mathrm{mg} / \mathrm{ml}$ ascorbic acid, and $100 \mathrm{mg} / \mathrm{ml}$ penicillin-streptomycin (Gibco; Thermo Fisher Scientific, Inc.), and incubated at $37^{\circ} \mathrm{C}$ in a humidified atmosphere containing $5 \% \mathrm{CO}_{2}$. The cells reached $80 \%$ confluence in 8-10 days, and were then suspended using trypsin-ethylenediaminetetraacetic acid, prior to passage for expansion. The cells from passage four were numerous and of suitable shape, and were used for subsequent cell transplantation.

To identify and track BMMCs following midpalatal transplantation, the cells were labeled with bromodeoxyuridine (BrdU; Sigma-Aldrich, St. Louis, MO, USA). The expanded and labeled BMMCs $\left(1 \times 10^{6}\right.$ cells $\left./ \mathrm{ml} ; 0.5 \mathrm{ml}\right)$ suspended in sterile medium were intra-orally injected into the left masseter area opposite the first molar in the EaT and EtR groups 2 days following the second activation, and the expansion appliances were maintained in place for two weeks. Physiological saline solution ( $5 \mathrm{mg} / \mathrm{kg}$; $0.9 \%$ sodium chloride) was injected, in the same manner, in the rats of the Exp and ExR groups.

Observation of histological changes in morphology. Once the rats had been sacrificed, the mandible and facial skin were removed. The maxillary tissue was fixed in $10 \%$ formalin solution [New England Biolabs (Beijing) Ltd., Beijing, China] for $48 \mathrm{~h}$, and was then demineralized in 10\% EDTA (pH 7.2; Sinopharm Chemical Reagent Co., Ltd., Beijing, China) for 6-8 weeks until the bone tissue had softened. Subsequently, samples of the midpalatal bone between the first and third molars of the rats were isolated and embedded with paraffin [New England Biolabs (Beijing) Ltd.]. Sections $(5 \mu \mathrm{m})$ were mounted on poly-L-lysine-coated glass slides [New England Biolabs (Beijing) Ltd.]. Prior to staining, the sections were incubated at $60^{\circ} \mathrm{C}$ for $1 \mathrm{~h}$, treated with xylene [New England Biolabs (Beijing) Ltd.], and rehydrated through a series of ethanol solutions. Hematoxylin and eosin [H\&E; New England Biolabs (Beijing) Ltd.] staining was performed to observe histological changes in morphology of the midpalatal bone and the surrounding tissues under an optical microscope (Olympus CX22; Olympus Corporation, Tokyo, Japan).

Tracking and evaluation of the differential potential of transplanted BMMCs in the masseter and midpalatal bone. The transplanted BMMCs labeled with BrdU were identified and tracked using immunofluorescence staining with mouse monoclonal anti-BrdU primary antibody (1:100; cat. no. B8434; Sigma-Aldrich). The paraffin sections were treated with 

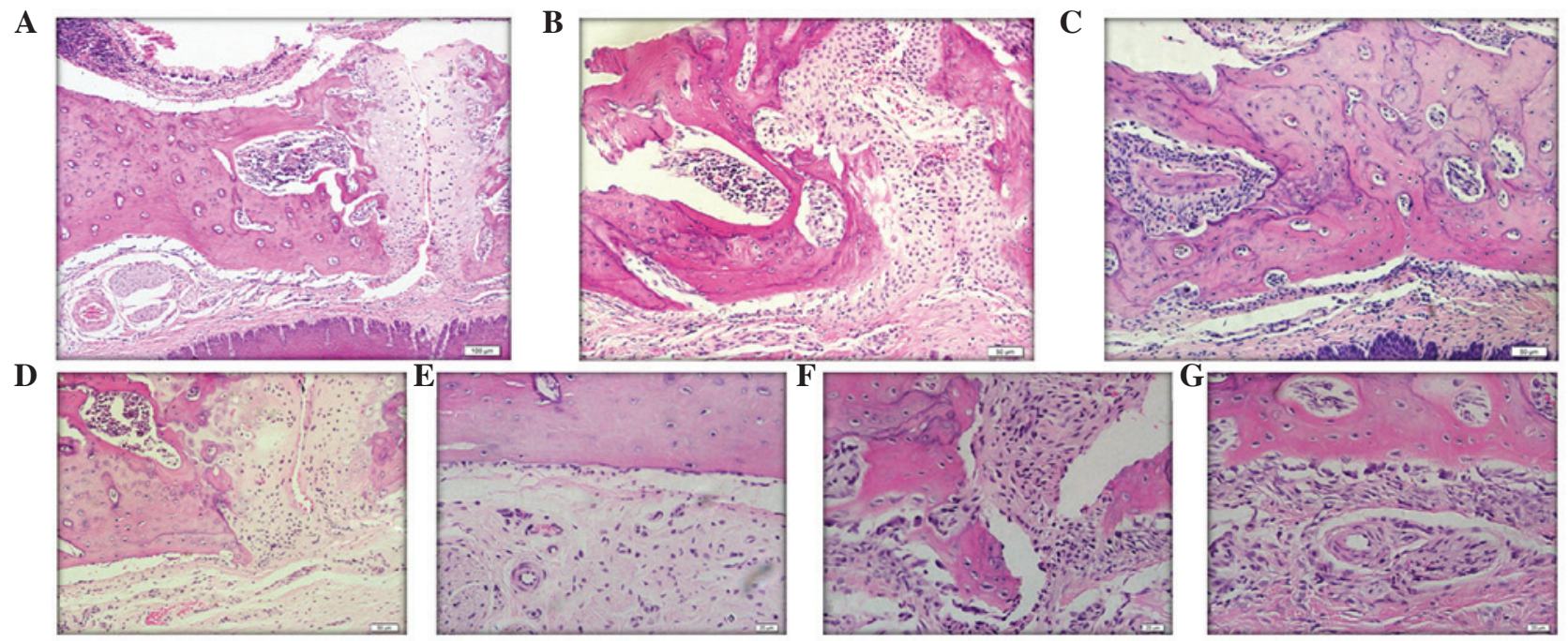

Figure 1. Microphotographs of the midpalatal bone and surrounding tissues following midpalatal expansion with or without BMMC transplantation in Sprague-Dawley rats. All images are oriented nasal side up and oral side down. (A) A central incision shown in the suture of the midpalatal bone in the Inc group. Arrows indicate bone-marrow-like cavity. Scale bar=100 $\mu \mathrm{m}$. (B) Central incision after 4 weeks, compared with the ExR group. Arrows indicate two layers of soft tissue and the mucosa at the nasal side. Scale bar=50 $\mu \mathrm{m}$. (C) BMMC transplantation accelerated midpalatal bone formation, exhibiting calcified compact bone in the EtR group. At 2 weeks following expansion in the Exp groups, the mesenchymal stem-like cells migrated from the soft tissue into the central area of the suture. Arrows indicate the mucosa at the oral side scale bar=50 $\mu \mathrm{m}$. In the (D) Exp group, the pattern of the chondrocyte array is evident. Arrows indicate a single layer of osteoblasts lining the oral and nasal surface of the bone. Scale bar=50 $\mu \mathrm{m}$. (E) A number of osteoblasts were present at the boundary of the midpalatal bone, and collagenic fibers were observed surrounding the neurovascular bundle in the Exp group. Arrows indicate a small number of endogenous spindle cells migrating from the submucosal tissue to the midpalatal suture. Scale bar=20 $\mu \mathrm{m}$. (F) In the EaT group, the majority of the chondrocytes had disappeared and were replaced by fibrous-like tissues, which contained numerous mesenchymal cells migrating from the submucosal tissue. Scale bar $=20 \mu \mathrm{m}$. (G) Submucosal layer was thicker and a large number of mesenchymal cells entered the surrounding compact palatal bone, transforming it into trabecular bone. Scale bar=20 $\mu \mathrm{m}$. BMMC, bone marrow mononuclear cells; Inc group, control group; Exp, expansion group; EaT, expansion and transplantation group; ExR, expansion and relapse group; EtR, expansion/transplantation/relapse group.

xylene and a series of ethanol solutions. Subsequently, antigen retrieval was conducted using $0.1 \%$ trypsin at $37^{\circ} \mathrm{C}$ for $5 \mathrm{~min}$, and the sections were incubated with $3 \% \mathrm{H}_{2} \mathrm{O}_{2}$ for $15 \mathrm{~min}$. The sections were then incubated with the antibody in a humid chamber at $4^{\circ} \mathrm{C}$ for $24 \mathrm{~h}$, prior to incubation with fluorescein isothionate (FITC) or tetramethyl-rhodamine-isothiocyanate (TRITC)-conjugated rabbit anti-mouse IgG secondary antibodies $(1: 100$; cat. no. YB-22908 and YB33709ES60, respectively; Shanghai Yubo Biotech Co., Ltd., Shanghai, China) at $37^{\circ} \mathrm{C}$ for $1 \mathrm{~h}$. The cell nuclei were visualized in the masseter and palatal tissues samples using 4',6-diamidino-2-phenylindole (DAPI; Molecular Probes, Thermo Fisher Scientific, Inc.). Fluorescence imaging was captured using a laser scanning confocal microscope (LSM710; Carl Zeiss AG, Oberkochen, Germany) or fluorescence microscope (Olympus BX61; Olympus Corporation) with an excitation wavelengths of $490 \mathrm{~nm}$ for FITC and $550 \mathrm{~nm}$ for TRITC. If the fluorescent signal areas (green) were consistent with the cell nuclei (blue) in the tissue samples stained with DAPI, the cells were determined to be BrdU-positive BMMCs.

$\mathrm{M}$-cadherin is a member of the $\mathrm{Ca}^{2+}$-dependent cell-cell adhesion molecule family, and its expression is confined to muscle satellite cells (19).Elivision ${ }^{\text {TM }}$ Plus kit(cat.no.KIT-9901) and Ultrasensitive ${ }^{\mathrm{TM}}$ S-P kit (cat. no. KIT-9709) (Fuzhou Maixin Biotechnology Co., Ltd., Fuzhou, China) were used to detect the expression of BrdU and M-cadherin (polyclonal rabbit anti-rat antibody; 1:100; cat. no. sc-10734; Santa Cruz Biotechnology, Inc., Dallas, TX, USA) in the masseter, in order to examine the distribution and myogenic orientation of the transplanted BMMCs. The blue/black BrdU-positive sections were visualized with 5-bromo-4-chloro-3-indolyl-phosphate (BCIP)/nitro-blue-tetrazolium, whereas the red/brown M-cadherin-positive sections were visualized with 3-amino-9-ethylcarbozole (AEC). The sections were counterstained with hematoxylin prior to being mounted.

The expression of osteocalcin by osteoblasts indicates the commencement of active bone formation (20). Double immunohistochemical staining for osteocalcin (polyclonal rabbit anti-rat antibody; 1:100; cat. no. BA0121-2; Wuhan Boster Biological Technology, Ltd., Wuhan, China) and BrdU were performed to investigate the osteogenic differentiation of the transplanted BMMCs in the palatal bone. The blue/black BrdU-positive sections were visualized with $\mathrm{BCIP/nitro-blue-tetrazolium,} \mathrm{whereas} \mathrm{the} \mathrm{red/brown} \mathrm{osteo-}$ calcin-positive sections were visualized with AEC.

\section{Results}

Changes of morphology following midpalatal expansion and $B M M C$ transplantation in rats. $\mathrm{H} \& \mathrm{E}$ staining was performed to observe the morphological changes of the midpalatal bone in the groups of rats. A palatal shelf structure with an incision in the middle of the suture is shown in Fig. 1A. The midpalatal suture was centrally located, and the hyaline cartilage was separated. The chondrocytes on either side of the suture gradually matured and migrated laterally towards the bone-marrow-like cavities and the compact bone of the maxilla. The bony plates were enveloped by two layers of soft tissue, mucosa at the nasal and oral sides. In the submucosa tissue on the oral side, the palatine vessels and nerves formed 
A

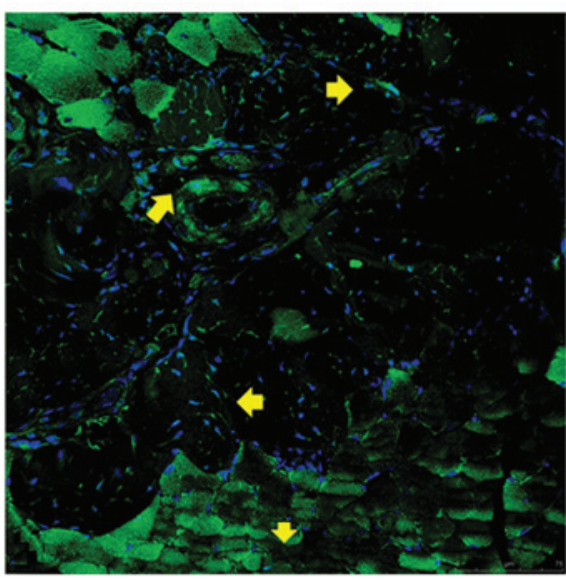

$\mathbf{D}$

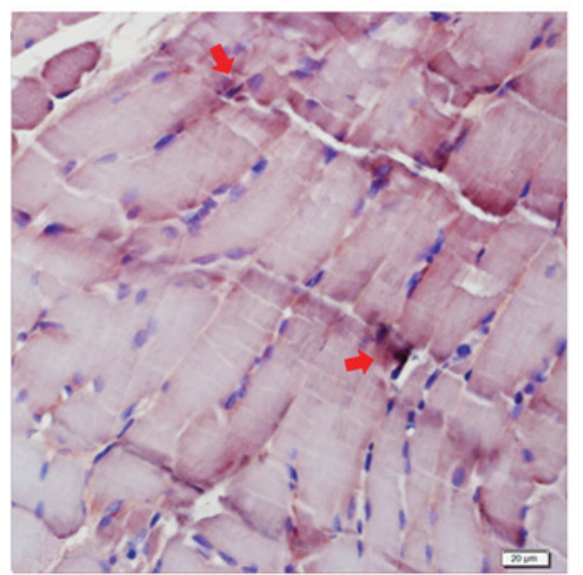

B

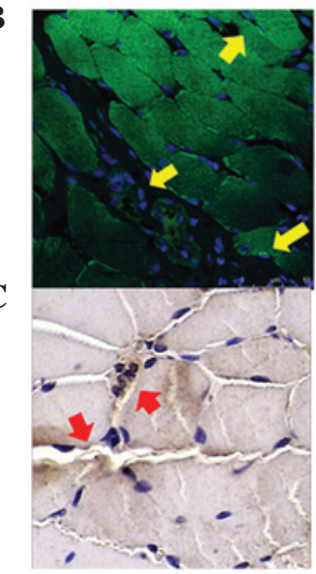

$\mathbf{E}$

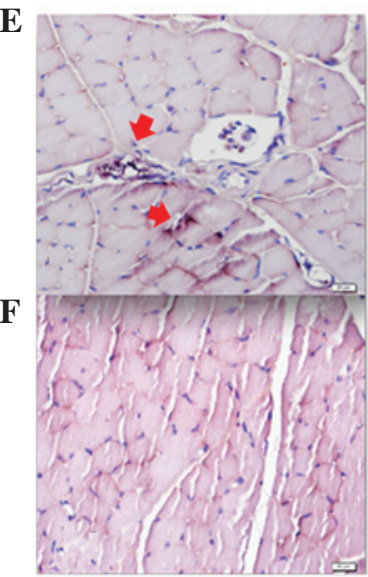

Figure 2. Microphotographs of immunohistochemical staining for BrdU-positive cells in the masseter area in Sprague-Dawley rats following expansion, with or without BMMC transplantation. (A and B) In the EaT group, the transplanted BMMCs predominantly appeared as spindle cells located in the peri-vessel sites and on the walls of the vessels. Arrows indicate BrdU-positive areas. Scale bar=75 and $50 \mu \mathrm{m}$, respectively. (C) In the EtR group, the cells were also seen penetrating into the vessels and exhibited a rounded morphology. Arrows indicate blue/black BrdU-positive staining. Scale bar=50 $\mu \mathrm{m}$. In the (D) EaT and (E) EtR groups, co-expression of BrdU and M-cadherin BMMCs was observed in the sub-laminar position, usually occupied by satellite cells. Arrows indicate blue/black BrdU-positive staining and red M-cadherin-positive staining. Scale bar=20 $\mu \mathrm{m}$. (F) No BrdU-positive cells were observed in the Exp group. Scale bar=20 $\mu \mathrm{m}$. BMMC, bone marrow mononuclear cells; BrdU, bromodeoxyuridine; Inc group, control group; Exp, expansion group; EaT, expansion and transplantation group; ExR, expansion and relapse group; EtR, expansion/transplantation/relapse group.

large bundles, which were located in bilateral concavities in the bone. A single layer of osteoblasts lined the oral and nasal surface of the bone. There were a small number of endogenous spindle cells migrating from the submucosa tissue into the midpalatal suture.

Following 2 weeks of expansion and relapse in the ExR group (Fig. 1B), the midpalatal suture was almost bridged by a large number of mononuclear cells. The chondrocytes remained evident and were involved in endochondral-type bone formation. By contrast, 4 weeks following BMMC transplantation in the EtR group (Fig. 1C), the suture and surrounding tissues had already constituted newly formed bone, with a calcified compact structure and several small vessels. In the Exp group, 2 weeks following expansion (Fig. 1D), endogenous spindle mesenchymal cells had migrated along the disconnected surface at the midline of the midpalatal suture. The chondrocytes were located laterally as mesenchymal cells, migrating into the incision ends and bridging one third of the incision. Following mechanical stimulation, the trabecular bone lining cells developed ultrastructural features of differentiated osteoblasts, and exhibited a cuboidal shape and rounded nuclei (Fig. 1E). By contrast, the majority of the chondrocytes in the EaT group disappeared and were replaced by fibrous-like tissues, which contained numerous mesenchymal cells migrating from the submucosal tissue (Fig. 1F). The submucosal layer became thicker, and numerous mesenchymal cells entered the surrounding compact palatal bone, which was transformed into trabecular bone (Fig. 1G).

Tracking and observation of the expression of $M$-cadherin of transplanted BMMCs in the masseter. During the 4 week experimental period, the BMMCs injected into the masseter were observed at several anatomical locations, traced by BrdU-positive staining. A number of positive spindle cells were located on the collagen fibers surrounding the blood vessels between the muscle fibers in the EaT group (Fig. 2A and B). During the experiment, a number of transplanted BMMCs penetrated into the blood vessels and exhibited rounded morphology in the EtR group (Fig. 2C). Co-expression of BrdU and M-cadherin was observed, which appeared in the sublaminar position, usually occupied by satellite cells (Fig. 2D, EaT group; Fig. 2E, EtR group). However, no BrdU-positive cells were observed in the Exp group (Fig. 2F). The observations demonstrated that the connective tissue fibers provided anchors 
A

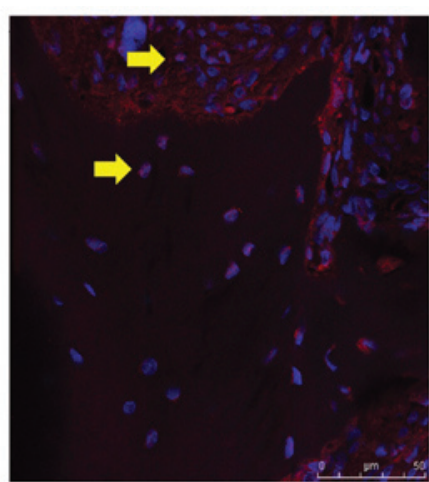

D

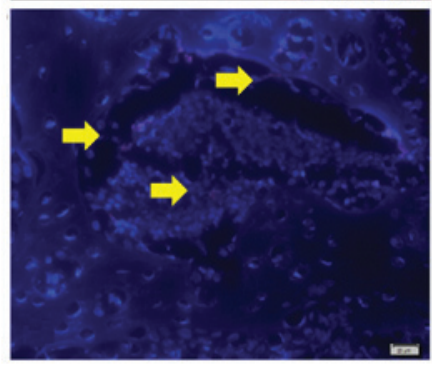

B

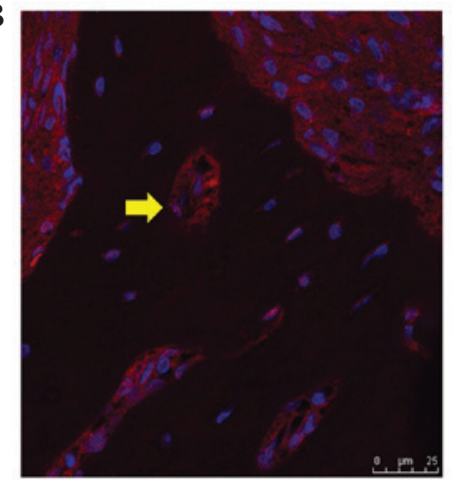

$\mathbf{E}$

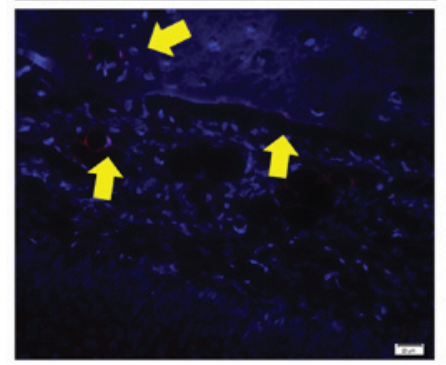

C

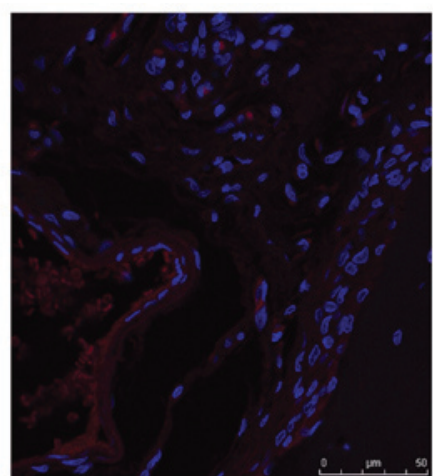

$\mathbf{F}$

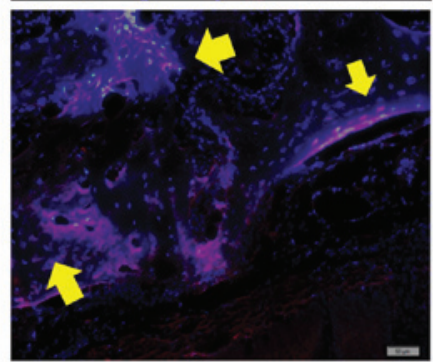

Figure 3. Immunofluorescent staining for BrdU in the midpalatal suture area in Sprague-Dawley rats following expansion with or without BMMC transplantation. (A) In the EaT group, as indicated by the arrows, the transplanted BMMCs were present in the suture area. Scale bar=50 $\mu \mathrm{m}$. (B) In the EtR group, the transplanted BMMCs appeared on the vessel walls in the midpalatal bone, as indicated by the arrows. Scale bar=25 $\mu \mathrm{m}$. (C) Mesenchymal-like cells were also present in the ExR group, and were involved in midpalatal bone remolding. Scale bar=50 $\mu \mathrm{m}$. (D) In the EaT group, as indicated by the arrows, the transplanted BMMCs were present in the bone marrow-like tissues. Scale bar $=20 \mu \mathrm{m}$. (E) Connective tissues surrounding the midpalatal bone provided a micro-platform for the stem cells in the EaT group. Scale bar=20 $\mu \mathrm{m}$. (F) In the EtR group, as indicated by the arrows, the transplanted BMMCs indicated by the blue-black BrdU-positive areas were differentiated into the pericytes of the neuromuscular bundles, and subsequently into osteoblasts, finally migrating into the midpalatal bone. Scale bar $=50 \mu \mathrm{m}$. BrdU, bromodeoxyuridine;BMMC, bone marrow mononuclear cells; EaT, expansion and transplantation group; ExR, expansion and relapse group; EtR, expansion/transplantation/relapse group.

A

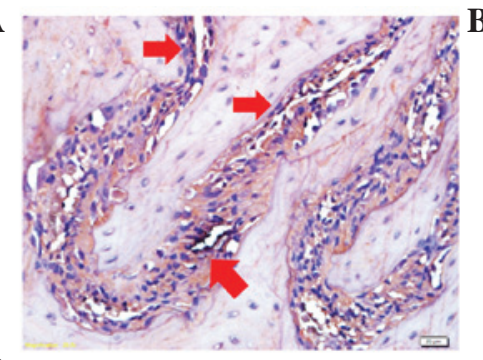

D

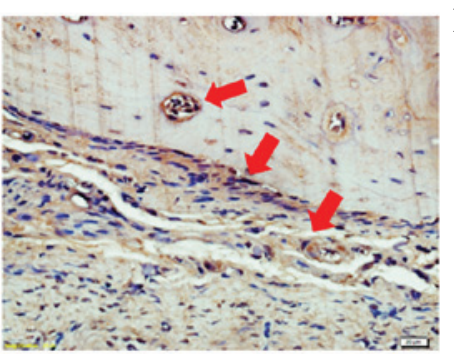

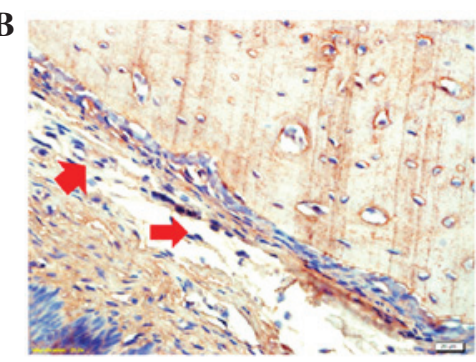

$\mathbf{E}$

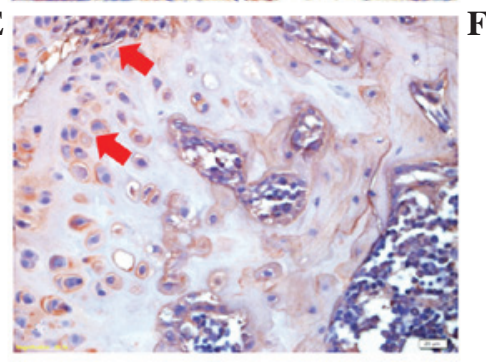

C

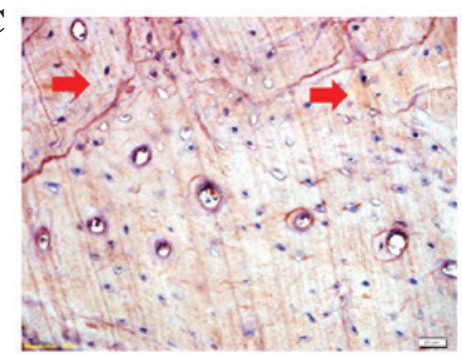

F

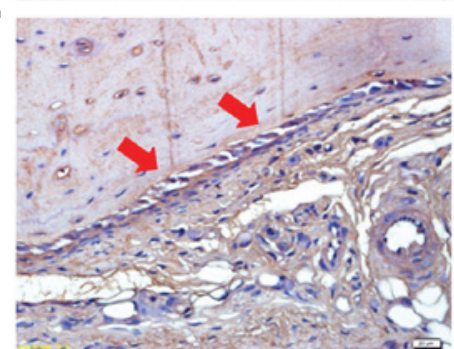

Figure 4. Co-expression of BrdU and osteocalcin in transplanted BMMC in the midpalatal bone and surrounding tissues in Sprague-Dawley rats following midpalatal expansion. (A) Transplanted BMMCs expressing osteocalcin are indicated by blue/black and red/brown color in the progenitor migrating channel, inn the submucosal tissue at the oral side, and on the (B) blood vessel walls (arrows) in the EaT group. (C) A small number of BrdU-positive BMMCs were located in the midpalatal bone in the EtR group, exhibiting a red-brown color. (D) Transplanted BMMCs expressing osteocalcin were observed at the junction of the mucosa and the midpalatal bone, demonstrating their transition from mesenchymal stem-like cells to osteoblasts. The blood vessel walls also contained BrdU and osteocalcin co-expressing transplanted BMMCs in the EtR group. (E) In the Exp group, no cell channels were observed, however, (F) a row of cubic oseoblasts arrayed orderly at the boundary were observed between the neuromuscular bundle and the midpalatal bone. Scale bar=20 $\mu \mathrm{m}$. BrdU, bromodeoxyuridine; BMMC, bone marrow mononuclear cells; Exp, expansion group; EaT, expansion and transplantation group; ExR, expansion and relapse group; EtR, expansion/transplantation/relapse group.

for the transplanted BMMCs to migrate between muscle fibers. These observations suggested that the transplanted BMMCs injected into the masseter were able to migrate into the blood circulation and possessed systemic characteristics. 
Tracking BMMCs in the midpalatal bone. Heterologous BMMC transplantation via masseter injection resulted in a large quantity of spindle cells migrating into the midpalatal bone injured by incision and expansion (Fig. 3). These cells included, not only transplanted BMMCs, but also endogenous mesenchymal-like cells. In the EaT group, BrdU-positive cells were located in the fibrous-like tissue at the midline of the suture (Fig. 3A), whereas they resided in the blood vessel walls (Fig. 3B) in the EtR group. In addition, mesenchymal-like cells in the ExR group were involved in midpalatal bone remodeling (Fig. 3C). The junction between the soft and hard tissue manifested the importance of the differentiation of mesenchymal-like cells into osteoblasts. In the EaT group, BrdU-positive cells were also located in the bone-marrow-like tissues (Fig.3D). The results clearly showed that the transplanted BMMCs, which appeared in the oral mucosa, enveloped the midpalatal bone in the EaT group (Fig. 3E), and these differentiated into osteoblasts at the boundary of the neuromuscular bundle and in the newly forming bone during midpalatal bone regeneration (Fig. 3F).

Expression of osteocalcin in BMMCs transplanted through the masseter into the midpalatal bone. During midpalatal expansion in the BMMC transplantation (EaT) group, fibrous-like tissue channels formed from the submucosal tissues at the nasal side in the midpalatal bone. These channels contained a small number of clones of transplanted BMMCs, which appeared as blue/black BrdU-positive sections, and also contained a large number of spindle cells that originated from the host soft tissues. Several spindle cells expressing osteocalcin were indicated by a red/brown signal in these progenitor migrating channels (Fig. 4A). Similar transplanted cells were observed in the submucosal tissue at the oral sides, which exhibited a darkened color indicating expression of osteocalcin, and a small number of transplanted BMMCs were located in the vessel walls in the EaT group (Fig. 4B, arrows). After 4 weeks, a few transplanted BMMCs were located in the midpalatal bone in the EtR group, which exhibited a light red/brown color, indicating lower expression levels of osteocalcin (Fig. 4C). In this group, a small number of BMMCs continued to co-express BrdU and osteocalcin, and were present at the junction of the mucosa and the midpalatal bone, as well as the vessels in the midpalatal bone (Fig. 4D). Although mesenchyma-like cells, migrating from the submucosa at the nasal side, were also observed, no cell channels were observed in the Exp group (Fig. 4E). Chondroblasts were located along the mesial incision of the midpalatal bone, and a row of cubic oseoblasts were arrayed orderly at the boundary between the neuromuscular bundle and midpalatal bone in the Exp group (Fig. 4F). A similar observation was observed in the BMMC transplantation group.

\section{Discussion}

Healing from injury requires the activation and proliferation of stem cells for tissue repair (21). In the present study, a rat model exhibiting midpalatal incision and expansion was used to observe the trajectory of BMMCs following BMMC transplantation via masseter injection. The BMMCs injected into the masseter were observed, not only in the masseter, but also in the blood vessels and oral mucosa, and enveloped the midpalatal bone. A number of the BMMCs differentiated into osteoblasts at the boundary of the neuromuscular bundle, and were located in the newly formed bone during midpalatal bone regeneration.

Rapid midpalatal expansion is a common treatment solution for patients with a narrow maxillary dental arch (22). The results of previous studies have revealed the mechanism of midpalatal bone remodeling induced by mechanical expansion. Hall et al (23) reported that the re-establishment of a suture with the palatine bone margins was covered by newly formed cartilage in the oral side of a mice model. It has also been suggested that mesenchymal cells located on the inner side of the cartilaginous tissue proliferate and differentiate into osteoblasts when the suture is expanded $(20,24)$. The midpalatal expansion model used in the present study differed from the above-mentioned studies, as a surgical incision was made in the suture to simulate the repair mechanism of distraction osteogenesis.

Several studies have demonstrated that stem cell transplantation has a marked effect on promoting callus formation and in shortening the consolidation period $(25,26)$. The results of the present study demonstrated that, in the expansion groups, a natural healing process occurred, in which mesenchymal progenitors in the surrounding soft tissues migrated into the injured midpalatal bone and repaired the damaged bone by differentiating into chondroblasts and osteoblasts. BMMC transplantation accelerated midpalatal bone formation by increasing the number of mesenchymal stem cells, which differentiated into osteoblasts directly. Previous studies have suggested that transplanted BMMCs induce bone regeneration by enhancing the migration of host stem cells into injury sites in an endocrine manner $(5,27,28)$. These regenerative cells migrate from the surrounding soft tissues and replace the endochondral bone formation with intramembranous bone formation, possibly due blood supply being increased (29), resulting in a microenvironment that favored stem cell differentiation into osteoblasts, rather than chondroblasts, due to the augmentation in oxygen content $(30,31)$. These observations indicate the critical effect of microenvironmental cues on cell fate. However, tissues with non-specific stem cells may become more tissue-specific under the effect of environmental factors (32). Multipotent stem cells exist in all tissues and have the ability to migrate among tissues in response to certain chemical signals. The mobilization patterns of stem cells may follow the laws of 'near to distant' and 'concentrated to diluted' in relation to various chemokines.

The regeneration consequence is a result of the combination of donor and endogenous stem cell migration to the injured midpalatal bone, either through osteogenic differentiation directly or through the production of cytokines $(33,34)$. In the present study, BMMC transplantation via masseter injection presents a potential dynamic process to repair injured midpalatal bone. A number of the transplanted BMMCs appeared to be located in the sub-laminar position, usually occupied by satellite cells. In addition, certain transplanted BMMCs anchored and migrated along the collagenic fibers over large distances. These results are concordant with 
those of Laird et al (35) who demonstrated that the interstitial migration or active amoeboid movement of stem cells are induced by extravascular cues, and this migration occurs independently of blood flow. Frimberger et al (36) reported rapid motility of hematopoietic stem cells, directed migration to stromal cells and marked membrane modulation. In the present study, a number of the transplanted BMMCs transformed into vessel pericytes and had the ability to enter into the circulation, acquiring systemic characteristics. Following maxilla incision and stimulation by mechanical expansion, the stem cells were mobilized and migrated to the enveloped soft tissue of the midpalatal bone. The capillary wall and the membrane surrounding the vascular bundles served as delivery sites for the donor cells and other sources of host stem cells to reconstruct the midpalatal bone. These results suggested that BMMCs mobilized other host stem cells, which contributed to the replenishment of osteoblasts for regeneration of the injured bone.

Further investigation into the mechanisms underlying the migration of stem cells from surrounding tissues or the circulation during injury healing and diseases are required to develop alternative novel therapies. In inflamed regions, mesenchymal stem cells rapidly exit the blood by integrating into the endothelium and migrating through the endothelial barrier, prior to penetrating the basement membrane and invading the surrounding tissue (37). Tumor formation has been suggested to be the result of continuously healing wound (38). Experimental and clinical studies have revealed that bone marrow-derived progenitor cells can migrate into the circulation, incorporate into tumor microenvironments and contribute to the growth of various tumors $(33,39)$. Chemotactic cytokines released by the damaged tissues mobilize progenitor cells by establishing mechanisms from the bone marrow, which may also regulate the mobilization of tissue-specific stem cells via other signaling pathways (40). Undifferentiated tumor cells may enter the circulation and result in distant solid tumor formation when the balance of a stem cell niche in various tissues is disrupted. This suggests that malignant tumor formation may be a type of systemic disease, which exhausts all the stem cells that maintain the physiological activities and restorative capacity of organisms. Therefore, it may prove useful to use chemical cytokines, which mobilize repair cells throughout the whole body, as targets for treating malignant tumors $(41,42)$.

In conclusion, the results of the present study demonstrated that BMMCs were able to enter the circulation, migrate from the muscle to bone tissue and were subsequently involved in tissue injury healing. These stem cells may be useful in the treatment of several types of cancer due to stem cell mobilization in the whole body.

\section{Acknowledgements}

The present study was funded by the National Natural Science Foundation of China (grant no. 81070804), the Shandong Province Science and Technique Foundation of China (grant no. 2014GSF118093) and Hubei-MOST KLOS \& KLOBME (grant no. 201101). The authors would like to thank Professor Xiaofei Tang for her advice on the interpretation of the histochemical experimental results.

\section{References}

1. Levi B, James AW, Nelson ER, Peng M, Wan DC, Commons GW, Lee M, Wu B and Longaker MT: Acute skeletal injury is necessary for human adipose-derived stromal cell-mediated calvarial regeneration. Plast Reconstr Surg 127: 1118-1129, 2011.

2. Ren G, Chen X, Dong F, Li W, Ren X, Zhang Y and Shi Y: Concise review: Mesenchymal stem cells and translational medicine: Emerging issues. Stem Cells Transl Med 1: 51-58, 2012.

3. Claes L, Recknagel S and Ignatius A: Fracture healing under healthy and inflammatory conditions. Nat Rev Rheumatol 8: 133-143, 2012.

4. Doherty MJ, Ashton BA, Walsh S, Beresford JN, Grant ME and Canfield AE: Vascular pericytes express osteogenic potential in vitro and in vivo. J Bone Miner Res 13: 828-838, 1998.

5. Khosla S, Westendorf JJ and Mödder UI: Concise review: Insights from normal bone remodeling and stem cell-based therapies for bone repair. Stem Cells 28: 2124-2128, 2010.

6. Phinney DG, Kopen G, Isaacson RL and Prockop DJ: Plastic adherent stromal cells from the bone marrow of commonly used strains of inbred mice: Variations in yield, growth, and differentiation. J Cell Biochem 72: 570-585, 1999.

7. Stamm C, Westphal B, Kleine HD, Petzsch M, Kittner C Klinge $\mathrm{H}$, Schümichen $\mathrm{C}$, Nienaber $\mathrm{CA}$, Freund $\mathrm{M}$ and Steinhoff G: Autologous bone-marrow stem-cell transplantation for myocardial regeneration. Lancet 361: 45-46, 2003.

8. Yamada M, Kubo H, Kobayashi S, Ishizawa K, Numasaki M, Ueda S, Suzuki T and Sasaki H: Bone marrow-derived progenitor cells are important for lung repair after lipopolysaccharide-induced lung injury. J Immunol 172: 1266-1272 2004

9. Parr AM, Tator $\mathrm{CH}$ and Keating A: Bone marrow-derived mesenchymal stromal cells for the repair of central nervous system injury. Bone Marrow Transplant 40: 609-619, 2007.

10. Strauer BE, Brehm M, Zeus T, Köstering M, Hernandez A, Sorg RV, Kögler G and Wernet P: Repair of infarcted myocardium by autologous intracoronary mononuclear bone marrow cell transplantation in humans. Circulation 106: 1913-1918, 2002.

11. Fodor WL: Tissue engineering and cell based therapies, from the bench to the clinic: The potential to replace, repair and regenerate. Reprod Biol Endocrinol 1: 102, 2003.

12. Chanda D, Kumar S and Ponnazhagan S: Therapeutic potential of adult bone marrow-derived mesenchymal stem cells in diseases of the skeleton. J Cell Biochem 111: 249-257, 2010.

13. Fong EL, Chan CK and Goodman SB: Stem cell homing in musculoskeletal injury. Biomaterials 32: 395-409, 2011.

14. Sundelacruz S and Kaplan DL: Stem cell- and scaffold-based tissue engineering approaches to osteochondral regenerative medicine. Semin Cell Dev Biol 20: 646-655, 2009.

15. Hatch HM, Zheng D, Jorgensen ML and Petersen BE: SDF-1alpha/CXCR4: A mechanism for hepatic oval cell activation and bone marrow stem cell recruitment to the injured liver of rats. Cloning Stem Cells 4: 339-351, 2002.

16. Lee K, Sugiyama H, Imoto S and Tanne K: Effects of bisphosphonate on the remodeling of rat sagittal suture after rapid expansion. Angle Orthod 71: 265-273, 2001.

17. Tuli R, Seghatoleslami MR, Tuli S, Wang ML, Hozack WJ, Manner PA, Danielson KG and Tuan RS: A simple, high-yield method for obtaining multipotential mesenchymal progenitor cells from trabecular bone. Mol Biotechnol 23: 37-49, 2003.

18. Haynesworth SE, Baber MA and Caplan AI: Cytokine expression by human marrow-derived mesenchymal progenitor cells in vitro: Effects of dexamethasone and IL-1 alpha. J Cell Physiol 166: 585-592, 1996.

19. Hsiao SP and Chen SL: Myogenic regulatory factors regulate M-cadherin expression by targeting its proximal promoter elements. Biochem J 428: 223-233, 2010.

20. Kobayashi ET, Hashimoto F, Kobayashi Y, Sakai E, Miyazaki Y, Kamiya T, Kobayashi K, Kato Y and Sakai H: Force-induced rapid changes in cell fate at midpalatal suture cartilage of growing rats. J Dent Res 78: 1495-1504, 1999.

21. Shi Y, Hu G, Su J, Li W, Chen Q, Shou P, Xu C, Chen X, Huang Y, Zhu Z, et al: Mesenchymal stem cells: A new strategy for immunosuppression and tissue repair. Cell Res 20: 510-518, 2010.

22. Haas AJ: The treatment of maxillary deficiency by opening the midpalatal suture. Angel Orthod 35: 200-217, 1965.

23. Hall B, Andreeff M and Marini F: The participation of mesenchymal stem cells in tumor stroma formation and their application as targeted-gene delivery vehicles. Handb Exp Pharmacol: 263-283, 2007. 
24. Takahashi I, Mizoguchi I, Nakamura M, Sasano Y, Saitoh S, Kagayama M and Mitani H: Effects of expansive force on the differentiation of midpalatal suture cartilage in rats. Bone 18 : 341-348, 1996

25. Kitoh H, Kitakoji T, Tsuchiya H, Mitsuyama H, Nakamura H, Katoh $\mathrm{M}$ and Ishiguro N: Transplantation of marrow-derived mesenchymal stem cells and platelet-rich plasma during distraction osteogenesis-a preliminary result of three cases. Bone 35: 892-898, 2004.

26. Qi M, Hu J, Zou S, Zhou H and Han L: Mandibular distraction osteogenesis enhanced by bone marrow mesenchymal stem cells in rats. J Craniomaxillofac Surg 34: 283-289, 2006.

27. Bi B, Schmitt R, Israilova M, Nishio H and Cantley LG: Stromal cells protect against acute tubular injury via an endocrine effect. J Am Soc Nephrol 18: 2486-2496, 2007.

28. Kumagai K, Vasanji A, Drazba JA, Butler RS and Muschler GF: Circulating cells with osteogenic potential are physiologically mobilized into the fracture healing site in the parabiotic mice model. J Orthop Res 26: 165-175, 2008

29. Matsumoto T, Kuroda R, Mifune Y, Kawamoto A, Shoji T, Miwa M, Asahara T and Kurosaka M: Circulating endothelial/skeletal progenitor cells for bone regeneration and healing. Bone 43: 434-439, 2008.

30. Zuscik MJ, Hilton MJ, Zhang X, Chen D and O'keefe RJ: Regulation of chondrogenesis and chondrocyte differentiation by stress. J Clin Invest 118: 429-438, 2008.

31. Colnot C, Zhang X and Knothe Tate ML: Current insights on the regenerative potential of the periosteum: Molecular, cellular and endogenous engineering approaches. J Orthop Res 30: $1869-1878,2012$.

32. Peng H and Huard J: Stem cells in the treatment of muscle and connective tissue diseases. Curr Opin Pharmacol 3: 329-333, 2003.

33. Iacovoni A, De Maria R and Gavazzi A: Alcoholic cardiomyopathy. J Cardiovasc Med (Hagerstown) 11: 884-892, 2010
34. Gharaibeh B, Lavasani M, Cummins JH and Huard J: Terminal differentiation is not a major determinant for the success of stem cell therapy-cross-talk between muscle-derived stem cells and host cells. Stem Cell Res Ther 2: 31, 2011.

35. Laird DJ, Von Andrian UH and Wagers AJ: Stem cell trafficking in tissue development, growth and disease. Cell 132: 612-630, 2008.

36. Frimberger AE, Mcauliffe CI, Werme KA, Tuft RA, Fogarty KE, Benoit BO, Dooner MS and Quesenberry PJ: The fleet feet of haematopoietic stem cells: Rapid motility, interaction and proteopodia. $\mathrm{Br}$ J Haematol 112: 644-654, 2001.

37. Steingen C, Brenig F, Baumgartner L, Schmidt J, Schmidt A and Bloch W: Characterization of key mechanisms in transmigration and invasion of mesenchymal stem cells. J Mol Cell Cardiol 44: 1072-1084, 2008

38. Park CC, Bissell MJ and Barcellos-Hoff MH: The influence of the microenvironment on the malignant phenotype. Mol Med Today 6: 324-329, 2000.

39. Roorda BD, Ter Elst A, Kamps WA and De Bont ES: Bone marrow-derived cells and tumor growth: Contribution of bone marrow-derived cells to tumor micro-environments with special focus on mesenchymal stem cells. Crit Rev Oncol Hematol 69: 187-198, 2009

40. Pitchford SC,Hahnel MJ,Jones CP and Rankin SM: Troubleshooting: Quantification of mobilization of progenitor cell subsets from bone marrow in vivo. J Pharmacol Toxicol Methods 61: 113-121, 2010.

41. Glass R, Synowitz M, Kronenberg G, Walzlein JH, Markovic DS, Wang LP, Gast D, Kiwit J, Kempermann G and Kettenmann H: Glioblastoma-induced attraction of endogenous neural precursor cells is associated with improved survival. J Neurosci 25: 2637-2646, 2005.

42. Walzlein JH, Synowitz M, Engels B, Markovic DS, Gabrusiewicz K, Nikolaev E, Yoshikawa K, Kaminska B, Kempermann G, Uckert W, et al: The antitumorigenic response of neural precursors depends on subventricular proliferation and age. Stem Cells 26: 2945-2954, 2008. 